\title{
Quark mass function from a OGE-type interaction in Minkowski space
}

\author{
Elmar P. Biernat* \\ CFTP, Instituto Superior Técnico, Universidade de Lisboa, Avenida Rovisco Pais 1, 1049 \\ Lisboa, Portugal, E-mail: elmar.biernatetecnico.ulisboa.pt
}

\section{Franz Gross}

Thomas Jefferson National Accelerator Facility (JLab), Newport News, Virginia 23606, USA and College of William and Mary, Williamsburg, Virginia 23188, USA

\section{T. Peña}

Departamento de Física and CFTP, Instituto Superior Técnico, Universidade de Lisboa, Avenida Rovisco Pais 1, 1049 Lisboa, Portugal

\section{Alfred Stadler}

Departamento de Física, Universidade de Évora, 7000-671 Évora, Portugal and CFTP, Instituto Superior Técnico, Universidade de Lisboa, Avenida Rovisco Pais 1, 1049 Lisboa, Portugal

The quark self-energy is calculated in both the spacelike and timelike regions of Minkowski space from an effective one-gluon-exchange and a constant interaction. The gauge dependence of the quark mass function is analysed and the model parameters are fixed by comparison of the results to the available lattice QCD data in the spacelike region.

Light Cone 2019 - QCD on the light cone: from hadrons to heavy ions - LC2019

16-20 September 2019

Ecole Polytechnique, Palaiseau, France

\footnotetext{
* Speaker.
} 


\section{Introduction}

The theoretical description of hadrons as bound states of QCD requires an essentially nonperturbative treatment. In particular, for the light mesons and the pion, the implementation of the low-energy phenomenon of dynamical chiral symmetry breaking $(\mathrm{D} \chi \mathrm{SB})$ is indispensable. In this work we report on recent results on the quark mass function in the Covariant Spectator Theory (CST) [1]. The CST [2, 3] is a covariant non-perturbative approach based on quantum field theory in Minkowski space and it has already been applied successfully to quarks and mesons in a number of previous papers $[4,5,6,7,8,9,10,11,12,13,14,15,16]$. It uses a covariant quarkquark interaction kernel that consists of Lorentz vector one-gluon-exchange (OGE) interaction, a generalized constant interaction, and a scalar-pseudoscalar generalization of a linear confining potential that is consistent with $\mathrm{D} \chi \mathrm{SB}$. In the non-relativistic limit, the covariant kernel reduces to the well-known "Cornell" potential and is therefore suitable for all mesons.

\section{CST quark self-energy}

In CST, the quark self-energy $\Sigma$ (times a renormalization constant $Z_{2}$ ) is given by the 3dimensional covariant integral [1]

$$
Z_{2} \Sigma(\not p) \equiv \frac{M\left(p^{2}\right)}{Z\left(p^{2}\right)}-m_{0}+\left[1-\frac{1}{Z\left(p^{2}\right)}\right] \not p=\frac{Z_{2}^{2}}{2} \sum_{\sigma= \pm} \int \frac{\mathrm{d}^{3} \mathbf{k}}{(2 \pi)^{3}} \frac{m}{E_{k}} \mathscr{V}\left(p, \hat{k}_{\sigma}\right)\left(\frac{m+\hat{k}_{\sigma}}{2 m}\right),
$$

where $M$ is the quark mass function, $Z$ the wave function normalization, $m_{0}$ denotes the bare quark mass, $\sigma= \pm$ labels the positive- and negative-energy on-shell momenta (corresponding to the positions of the quark propagator poles at $\left.k^{2}=m^{2}\right)$, and $\hat{k}_{\sigma}=\left(\sigma E_{k}, \mathbf{k}\right)$ with $E_{k}=\sqrt{m^{2}+\mathbf{k}^{2}}$. The invariant functions $M$ and $Z$ defined in Eq. (2.1) satisfy the real mass-pole conditions at $p^{2}=m^{2}$ :

$$
M\left(m^{2}\right)=m \quad \text { and } \quad Z\left(m^{2}\right)=Z_{2}\left[1-\left.2 m \frac{\mathrm{d} M\left(p^{2}\right)}{\mathrm{d} p^{2}}\right|_{p^{2}=m^{2}}\right] .
$$

The interaction kernel is given by

$$
\begin{aligned}
\mathscr{V}\left(p, \hat{k}_{\sigma}\right)= & \frac{1}{4} \sum_{a=1}^{8} \lambda_{a} \otimes \lambda_{a}\left\{\left(\mathbf{1} \otimes \mathbf{1}+\gamma^{5} \otimes \gamma^{5}\right) V_{\ell}\left(p, \hat{k}_{\sigma}\right)\right. \\
& \left.-\gamma_{\mu} \otimes \gamma_{v}\left[\mathrm{~g}^{\mu \nu}-(1-\xi) \frac{q_{\sigma}^{\mu} q_{\sigma}^{v}}{q_{\sigma}^{2}}\right]\left[V_{g}\left(p, \hat{k}_{\sigma}\right)+V_{c}\left(p, \hat{k}_{\sigma}\right)\right]\right\} .
\end{aligned}
$$

Here $V_{\ell}$ is a covariant generalization of the linear confining potential [13], $\xi$ is a parameter specifying the linear covariant gauge, $q_{\sigma}=p-\hat{k}_{\sigma}$,

$$
V_{g}\left(p, \hat{k}_{\sigma}\right)=\frac{4 \pi \eta \alpha_{s} g(y)}{M_{g}^{2}+\left|q_{\sigma}^{2}\right|}
$$

is an OGE-type interaction with $\eta$ a mixing parameter (discussed below), $\alpha_{s}$ the unrenormalized strong coupling constant, $g(y)$ a regularization form factor depending on the covariant variable $y^{2}=\frac{\left(p \cdot \hat{k}_{\sigma}\right)^{2}}{p^{2} \hat{k}_{\sigma}^{2}}$, and

$$
V_{c}\left(p, \hat{k}_{\sigma}\right)=\frac{(1-\eta) C E_{k}}{2 m}(2 \pi)^{3} \delta^{3}\left(\mathbf{k}-\frac{m}{\sqrt{p^{2}}} \mathbf{p}\right) h\left(p^{2}\right)
$$


is a covariant form of a constant potential with the unrenormalized strength $C$ and $h\left(p^{2}\right)$ is a strong quark form factor normalized as $h\left(m^{2}\right)=1$. The OGE and constant parts are both assumed to be vector interactions with the same gauge dependence. This is because the constant kernel can be regarded as part of - or a correction to-the OGE part, with their respective effective strengths related by the mixing parameter $\eta$ and chosen such that always the same constituent quark mass $m$ is generated through Eq. (2.2) [1]. Furthermore, in the OGE kernel, Eq. (2.4), a finite gluon mass $M_{g}=0.6 \mathrm{GeV}$ has been introduced, and the prescription $q_{\sigma}^{2} \rightarrow-\left|q_{\sigma}^{2}\right|$ has been applied to remove the unwanted singularity in the gluon propagator [1]. In addition, both the large- $\mathbf{k}^{2}$ and small$p^{2}$ singular behaviors of the OGE contribution are regularized by an appropriately-chosen form factor $g(y)$, which introduces a cutoff parameter $\Lambda_{g}$. Finally, recall that $V_{\ell}$ with a Lorentz scalarpseudoscalar structure does not contribute to the self-energy [11], which simplifies the calculation considerably.

\section{Results and Conclusion}

The main result of this work is the quark mass function calculated in the chiral limit $\left(m_{0}=0\right)$ and for 3 different linear covariant gauges ( $\xi=0,1,3)$, as summarized in Fig. 1.
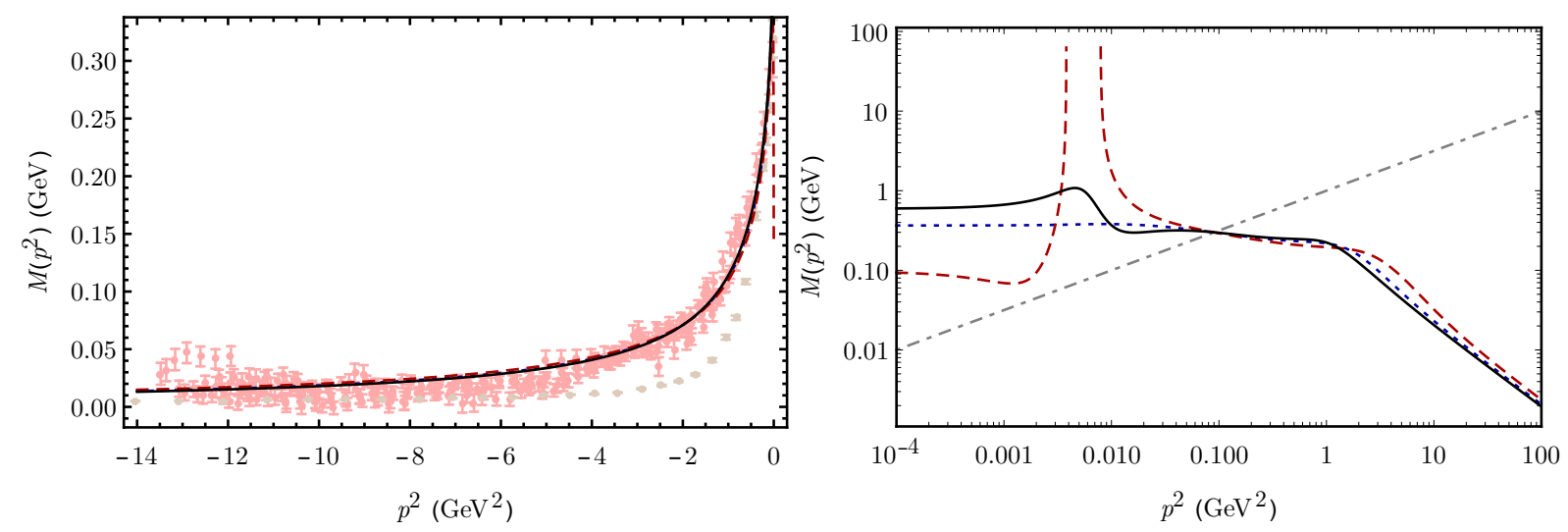

Figure 1: The mass function (in $\mathrm{GeV}$ ) vs. $p^{2}$ (in $\mathrm{GeV}^{2}$ ) for $\xi=0$ and $\Lambda_{g}=0.9 \mathrm{GeV}$ (red dashed line), $\xi=1$ and $\Lambda_{g}=0.6 \mathrm{GeV}$ (blue dotted line), and $\xi=3$ and $\Lambda_{g}=0.45 \mathrm{GeV}$ (black solid line). The left panel shows the spacelike region with lattice QCD data taken from [17] (red data points) and [18] (brown data points). The right panel shows the timelike region on logarithmic scales where the intersection of the curves with $\sqrt{p^{2}}$ (gray dotdashed line) marks the $\xi$-independent on-shell point $p^{2}=m^{2}$ defining the chiral constituent quark mass.

We have chosen a chiral constituent quark mass of $m=0.3 \mathrm{GeV}$ and an effective renormalized OGE strength $\eta Z_{2}^{2} \alpha_{s}=0.5$. The cutoff parameter $\Lambda_{g}$ was then roughly adjusted, separately for each gauge, to agree with the lattice QCD data [17]. The Landau gauge $(\xi=0)$ result has a pole at some small timelike $p^{2}$. Such poles are not present in the Feynman $(\xi=1)$ and Yennie $(\xi=3)$ gauges. Further, it should be mentioned that, as a consequence of the prescription to make the dressed gluon propagator non-singular, the mass function develops a discontinuity at $p^{2}=0$ (not displayed in the figure). The discontinuity depends on the gauge, and we find that the quark mass function is continuous at $p^{2}=0$ only for the Yennie gauge, which therefore is the preferred gauge 
in this CST calculation. For timelike $p$ we find a strong dependence of the quark mass function on the gauge. However, on-mass-shell quantities, such as the constituent quark mass are gaugeparameter independent - a nice feature of our CST approach that has been proven in Ref. [1]. For spacelike $p$ the gauge dependence is very weak, which allowed us to use the existing lattice QCD data to adjust the model parameters. After extending the present calculation to finite bare quark masses, the mass functions can then be used in meson calculations, thereby achieving complete consistency between the quark self-interaction and the quark-antiquark interaction.

\section{Acknowledgments}

This work was funded in part by Fundação para a Ciência e a Tecnologia (FCT) under Grants No. CFTP-FCT (UID/FIS/00777/2013 and UID/FIS/00777/2019), No. SFRH/BPD/100578/2014, and No. SFRH/BD/92637/2013. E.B. was supported by FCT and IST-ID under contract No. ISTID/148/2018 and F.G. was supported by the U.S. Department of Energy, Office of Science, Office of Nuclear Physics under contract DE-AC05-06OR23177.

\section{References}

[1] E. P. Biernat, F. Gross, M. T. Peña, A. Stadler, and S. Leitão, Phys. Rev. D 98, 114033 (2018).

[2] F. Gross, Phys. Rev. 186, 1448 (1969).

[3] F. Gross, Phys. Rev. C 26, 2203 (1982).

[4] F. Gross and J. Milana, Phys. Rev. D 43, 2401 (1991).

[5] F. Gross and J. Milana, Phys. Rev. D 45, 969 (1992).

[6] F. Gross and J. Milana, Phys. Rev. D 50, 3332 (1994).

[7] Ç. Şavklı and F. Gross, Phys. Rev. C 63, 035208 (2001).

[8] E. P. Biernat, F. Gross, M. T. Peña, and A. Stadler, Phys. Rev. D 89, 016005 (2014).

[9] E. P. Biernat, F. Gross, M. T. Peña, and A. Stadler, Phys. Rev. D 89, 016006 (2014).

[10] E. P. Biernat, F. Gross, M. T. Peña, and A. Stadler, Phys. Rev. D 92, 076011 (2015).

[11] E. P. Biernat, M. T. Peña, J. E. Ribeiro, A. Stadler, and F. Gross, Phys. Rev. D 90, 096008 (2014).

[12] E. P. Biernat, F. Gross, T. Peña, and A. Stadler, Few-Body Syst. 54, 2283 (2013).

[13] S. Leitão, A. Stadler, M. T. Peña, and E. P. Biernat, Phys. Rev. D 96, 074007 (2017).

[14] S. Leitão, A. Stadler, M. Peña, and E. P. Biernat, Phys. Lett. B 764, 38 (2017).

[15] S. Leitão et al., Eur. Phys. J. C 77, 696 (2017).

[16] S. Leitão, A. Stadler, M. T. Peña, and E. P. Biernat, Phys. Rev. D 90, 096003 (2014).

[17] P. O. Bowman et al., Phys. Rev. D 71, 054507 (2005).

[18] O. Oliveira, P. J. Silva, J.-I. Skullerud, and A. Sternbeck, Phys. Rev. D 99, 094506 (2019). 